\title{
UNFAVOURABLE EFFECT OF BLASTICIDIN-S ON HUMAN BODY WITH SPECIAL REFERENCE TO THE IMPAIRMENT OF WORKERS IN A PLANT PRODUCING BLASTICIDIN-S
}

\author{
Yukiko OHTA (Miss) \\ Graduate School of Social Medicine, University of Tokyo, Hongo, Bunkyo-ku, Tokyo
}

(Received July 10, 1963)

\begin{abstract}
Injurious effect of Blasticidin-S, the agricultural antibiotic effective against Piricuraria orizae, discovered in Japan was investigated in the production plant. The result of this investigation was reported and discussion was made on its protective measures. Blasticidin-S causes very severe, acute inflammation on the mucous membrane and epidermis exposed to the antibiotic. Among the injurious effects of Blasticidin-S, some marked symptoms: in the eyes, intestines, and skin were described. Results of investigations on subjective symptoms of the whole workers were listed and adverse effect of Blasticidin-S against human body was clarified. Measures against these injuries include, first, the prevention of contact with the antibiotic and then prevention of continuous exposure, when the worker is likely to be in contact with Blasticidin-S in minute amounts. These measures were found to lessen the degree of injury. Any injuries that have occurred have been alleviated by administration of antiinflammatory hormone and antibiotics. This seems to suggest measures against future injuries.
\end{abstract}

Blasticidin-S is produced in the culture medium of Streptomyces griseochromogenes Fukunaga and is an agricultural antibiotic discovered in Japan in 1951. According to Takeuchi $e t a l^{1}$ and Sakagami ${ }^{2}$, it is a basic substance obtained as white needle crystals, melting at $235 \sim 236^{\circ}$, molecular weight about $300 \sim 450$. Its structure has not been elucidated as yet but its analytical values correspond to the molecular formula of $\mathrm{C}_{14} \mathrm{H}_{20} \mathrm{O}_{5} \mathrm{~N}_{6}$. It does not contain sulfur, phosphorus and halogen. It is easily soluble in water and acetic acid, and insoluble in majority of organic solvents such as methanol, ethanol, acetone, benzene, ether, and chloroform. It is stable to heat and ultraviolet rays. Its hydrolysis is said to produce cytosine and uracil, the componental bases of nucleic acids, as well as N-methyl-6-amino-3-carbamoyl-2-piperidone and levulinic acid.

The organomercurials hitherto used for combatting Piricularia oryzae has a good effect on preventing this disease but has no therapeutic effect. Blasticidin-S has the effect of preventing this disease comparable to that of organomercurials but has additional effect of stopping the disease even after it has further advanced, and in much 
greater degree than the existing chemicals. While some of the foreign strains of rice plants, such as the Indian rice, were affected by organomercurials, Blasticidin-S has a great effect of preventing this disease without adverse effect on foreign strains. Therefore, much is expected of its production and demand in the future.

On the other hand, some unfavourable effect of this antibiotic was found among plant workers handling Blasticidin-S, particularly in eyes, skin, and digestive organs. It was therefore felt necessary and imperative to carry out studies on the fundamental action of Blasticiding-S on human body and to find whether its action would cause any chronic injuries or impairments. The present series of work were therefore undertaken to find the extent of ill effect on workers handling Blasticidin-S and a part of biological action of Blasticidin-S on human body was revealed. Examinations were also made to find preventive measures against such ill effects.

\section{Methods And Results}

Actual injuries to eyes, skin, and digestive system in workers during industrial production of Blasticidin-S were investigated. Animal experiments were also carried out with mice to examine the histological changes of critical organs in acute poisoning and whether chronic injuries or chronic poisoning might be produced in the manufacturing plant. The present paper reports only the incidence of injuries in the plant, and results of animal experiments will be reported elsewhere.

\section{Brief Outline of the Work Involved and Environmental Conditions}

Production of Blasticidin-S in the plant examined was made during 1961 and 1962, and the quantity produced in 1962 was about 10 times greater than that in 1961 . Therefore, the incidence of injuries to be described below also increased in 1962, although the plant was in experimental production with rather poor facilities at that time. Workers were made to wear protective garment and to use protective tools, but it is natural that many factors, which oppose to smooth development of a new work,

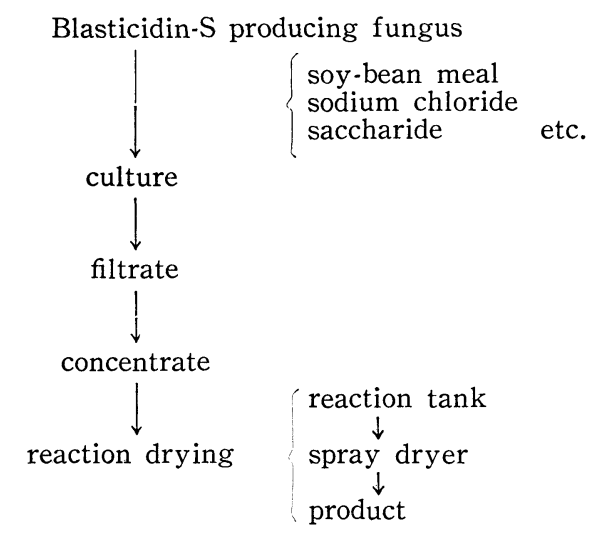

Fig. 1. Process of production of Blasticidin-S. 


\section{UNFAVOURABLE EFFECT OF BLASTICIDIN-S}

are present in the plants at the early stage of production. In this particular instance, many of the injurious effects observed seemed to be due to the defect in facilities rather than to insufficient experience of the workers.

The flow-chart of the production work is given in Fig. 1. Streptomyces griseochromogenes, which produces Blasticidin-S, is cultured in a medium, the culture medium is filtered to separate cell debris, and the filtrate is further purified. The concentrated filtrate is treated to crystallize the antibiotic out, the crystals are dried, weighed, and made into the product for market. The crystals of Blasticidin-S are fine and light, easily disperse into the air, therefore the majority of ill-effects occurred in the drying process. Age distribution of the workers in the area where majority of illeffects were found is shown in Fig. 2.

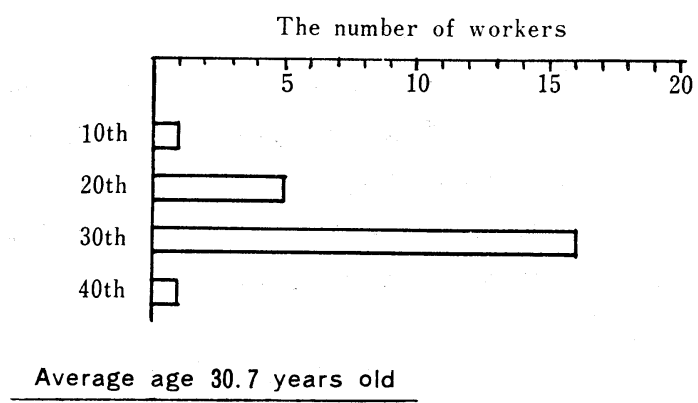

Fig. 2. Age distribution of workers.

\section{Ill Effects of Blasticidin-S at the Time of Examination}

Some examples of severe injuries found are listed below.

Case No. 1 ; T. H. Male, Ared 30

Had been in the research team for industrial production (including filtration, purification, concentration, and drying) of Blasticidin-S for about 3.5 years in the so-called Intermediate Test Room. Some ill-effects were noticed since December 24, 1959. Chief complaint was a rash in the abdominal to chest region, with strong itching. The rash appeared in various parts of the body from time to time, and conjunctivitis resulted from fine pieces of the antibiotic crystal taken in the eyes. Conjunctivitis was cured rather simply, but a serious injury on the eyes occurred on September 19, 1960. In this case, the worker was bottling the crystals. The room temperature was $32^{\circ} \mathrm{C}$ and the work was done with profuse perspiration. This worker wore eye glasses and, during wiping of the glasses, fine powder of the antibiotic might have entered the eyes. The eyes were washed immediately and the washing was continued until the worker became free from pain. He was allowed to go home without further treatment. During the night, he began to feel pain with secretion and was unable to open the eyes. He consulted an eye specialist and was diagnosed as acute conjunctivitis, without any abnormal findings in the fundus or cornea. Visual acuity was found below 0.1 
and marked congestion and inflammation of conjunctiva bulbi and palpebrae were also observed. The symptoms, however, alleviated remarkably after one week.

Case No. 2; M. K. Male, Aged 34

Had been in the research team making chemical experiments on the purification of Blasticidin-S. First incidence occurred on March 12, 1960. While handling the solution of Blasticidin-S in a test tube, some liquid entered the eyes but the symptoms disappeared after about three times of treatments. There were possibilities for the antibiotic to enter the eyes as he was always working with Blasticidin-S. Since then, the said eye injuries required physician's activity and consultations were made 7 to 22 times a month for each worker. Eye specialist diagnosed it as acute conjunctivitis, but this was also accompanied with blepharitis marginalis with eye-wax, blephanorrhoa, often required eye-washing once in every two hours. When the inflammation was at the worst, adrenocortical steroid eye-lotion was used but complete cure was not obtained. The work on Blasticidin-S was therefore interrupted since September 1961 and the symptoms became better. He visited the eye specialist in March, 1962. There were proved the stricture of lachrymal point as the sequelae of blepharitis marginalis, the turbidity and proliferation in marginal area of the cornea and post-inflammatory scar of the corea. These lesions have been cured now.

Case No. 3 ; Y. I. Male, Aged 37

Intermediate test work. In preliminary test of broth filtration, on December 23, 1961, he was watching the flow rate of the broth and the broth spurted out of the press frames entered his eyes. Diagnosis of the eye specialist was acute conjunctivitis with corneal ulceration and required 16 days of rest. Examination after about one month did not reveal the turbidity of the deep layer of the corea and scar resulting from the inflammation.

Besides the cases mentioned above, one man (K. N., aged 30) was laid down for 2 days because of acute conjunctivitis with epithelial erosion on January 31, 1961 ; one man (Z. T., aged 31) for 4 days due to acute conjunctivitis with keratitis on January 30, 1962 ; one man (K.S., aged 19) for 3 days due to acute conjunctivitis with keratitis on March 27, 1962. During 1961, four workers (including one female, aged 19) were laid down for 5 days with conjunctivitis.

Case No. 4 ; K. M. Male, Aged 30

Worked in the Intermediate Test Room. The most unfortunate case occurred on July 14, 1961, while K. M. and six others were working on drying test of Blasticidin-S with a spray dryer. It was in a very hot season, the room was extremely hot and humid as the dryer was working at $150 \sim 200^{\circ} \mathrm{C}$. On account of strong perspiration, the workers were in their undershirt except for protective goggles and protective respirators. Clusters of papule appeared on bare surface, on the face, arms, neck and chest. The site of rash became reddened and was accompanied by suppuration due to secondary bacterial infection due to sweating, dust, etc., suppuration corresponding to hair 


\section{UNFAVOURABLE EFFECT OF BLASTICIDIN-S}

follicles. Pain and itching were very severe and rash lasted for about one month, and the patient was treated for only about a week. The treatment was an application of ointment containing adrenocortical steroid and an antibiotic. On the other hand, one of these six workers was free from any skin rash although he had worked under the same conditions.

Urine tests (urobilinogen, bilirubin, blood corpuscles, and protein reaction) on the workers two days after the above episode showed no abnormality.

Case No. 5 ; M. T. Male, Aged 35

Worked in the Intermediate Test Room. The case showing the most severe diarrhoea. Slight diarrhoea had been experienced in the past but the amount of Blasticidin-S handled had been small and the cause could not be traced to it. $\mathrm{He}$ visited the factory clinic at the beginning of February, 1961, with the complaint of diarrhoea and received medical treatment several times thereafter. There was no abdominal pain but watery stool appeared 3 5 times a day. The most marked feature

Fifty-eight workers were examined. (From 1961 to 1962)

Eye Injury

Discharge form both eyes

Eye redness

Eye fatigue

Feeling more light sensation

Feeling foreign body in the eyes

Pain, anti-biting sensation

Tears flowing.

Itching of both lid margins

Skin Injury

Itching of skin arms; legs

Rash body

all exposure parts

Swelling redness

Nose Injury

Nose stopping up

Nose bleeding a little

Discharge from nose

Nose pain

Swelling in nose

Nose bleeding much

Injury of Digestive Organs

Diarrhoea

Watery stool from time to time

Diarrhoea with pain

Diarrhoea with uncomfortable

feeling but no pain

Throat Injury

Throat pain

Coughing

Choking with cigarette smoke

Having phlegms

Festering on injured skin

Fatigue

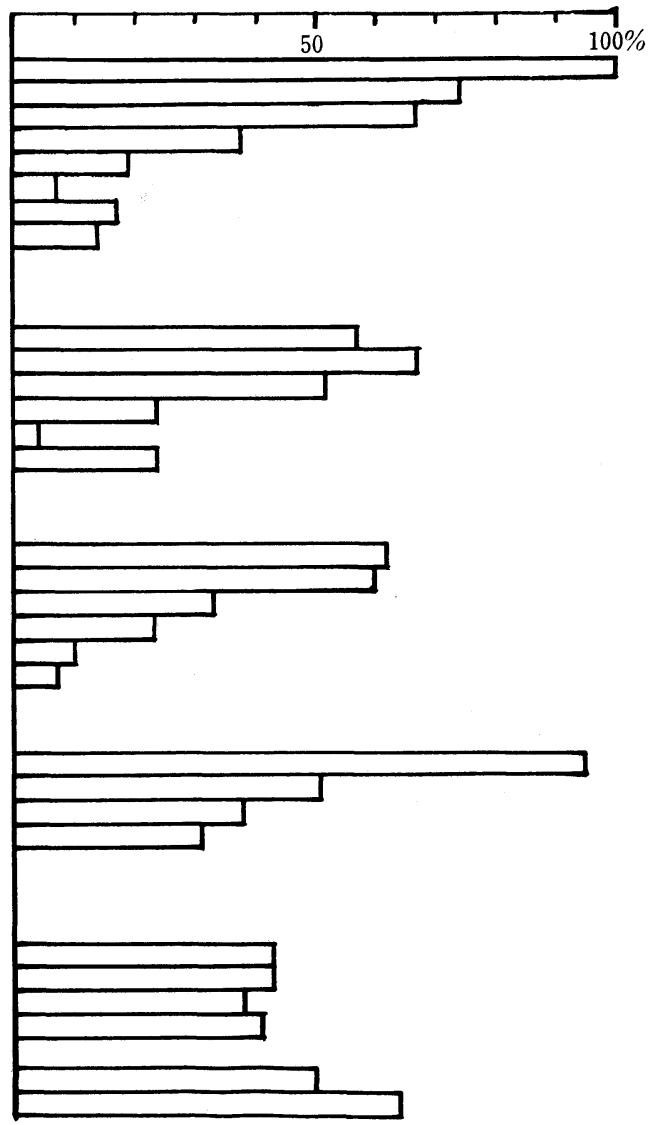

Fig. 3. Subjective symptoms. 


\section{Y. OHTA}

was a watery diarrhoeal discharge twice during the night of August 7, 1961, after working with Blasticidin-S from 2 to 3 o'clock in that afternoon. Another attack was on December 21, when he handled Blasticidin-S, and he had five times a day of diarrhoea started from the morning of December 22 .

The foregoing cases indicate the marked characteristic of the effect of Blasticidin$\mathrm{S}$, and the degree of injury differs from case to case. Investigations were made on clinical symptoms on all 58 workers working in the drying procedure three times in all, and the results are summarized in Fig. 3. The quantity of production during 1962 is 10 times to that in 1961 and the percentage of incidence might be greater than that given in Fig. 3.

Systemic Symptoms: Complaint of fatigue is the greatest. This is thought to be due chiefly to muscular work during the night, especially to work in the open, and lack of sleep, added with mental tension and diarrhoea.

Eye Injury: Complaints of eye injury have been made by all the workers and the injury extends over a wide range. Most of them have been cured after a slight attack of conjunctivitis. There were five cases of blepharitis marginalis and keratitis, but they have been also cured.

Injury of Digestive Organs: With the exception of one worker, all experienced. diarrhoea, which was characterized by frequent discharges, the most severe case having $9 \sim 10$ times a day. The feature of this diarrhoea is that it occurs suddenly with stool impulse but the diarrhoea stops spontaneously after one or two days. Abdominal pain at the time of discharge was felt in only five persons. There was no complaint of gastric disturbances except for chronic non-occupational disorder.

Skin Injury: Most of the work had been carried out during the winter and wearing of many clothes prevented sticking of Blasticidin-S powder directly on the skin. Even then, majority of the workers experienced rash in some part of the body. There was a strong itching and many of the workers complained of pain after finishing work and taking a bath. The rash was various, presenting urticaria, papule, and impetigo. Some showed allergic symptoms and the rash appeared on the back and inguinal region. If the antibiotic powder attached on injured skin, suppuration occurred and left a weal even after cure. There was one example in which the weal remained after work in April of 1961 and it had to be removed by plastic surgery. Suppuration generally occurred on injured skin, chaps, and roughened skin of hands and fingers.

Injuries on nose and throat: Almost all of the workers complained of nose bleeding. of a slight degree. In the throat, inflammation of soft palate was seen in many of the workers and two of them complained of persistent pain. Accompaniment of upper respiratory disorders was seen in some of them, during the winter a part of work being. carried out in the open air, and the work in the packing room being carried out at high temperature. There were some cases of upper respiratory inflammation due to the sulfur dioxide gas in the packing room. In such a case, the cause is not 


\section{UNFAVOURABLE EFFECT OF BLASTICIDIN-S}

only the antibiotic but many of the complaints seemed to be due to other causes. Complaint of coughing for $2 \sim 3$ weeks after stopping of the operation and of choking with cigarette smoke were also noted.

\section{Skin Patch Test}

For 16 days from December 4, 1961, skin patch test was carried out by placing an ointment of carbowax containing $10 \%$ of Blasticidin-S $(540 \mathrm{r} / \mathrm{mg})$ on the inside of upper arm and the effect was observed after 48 hours. The test was carried out on 13 workers, two being positive, 3 being false positive, and others were negative. However, negative cases developed reddening, blisters, and rash on the test spot one week after the test, and one of them developed rash in the applicated site.

These workers were exposed to Blasticidin-S during the first production in February, 1961, and might have shown allergic reaction if Blasticidin-S were antigenic, but nothing could be concluded from the above test so far. This test is only indicative of skin sensitivity to a certain degree.

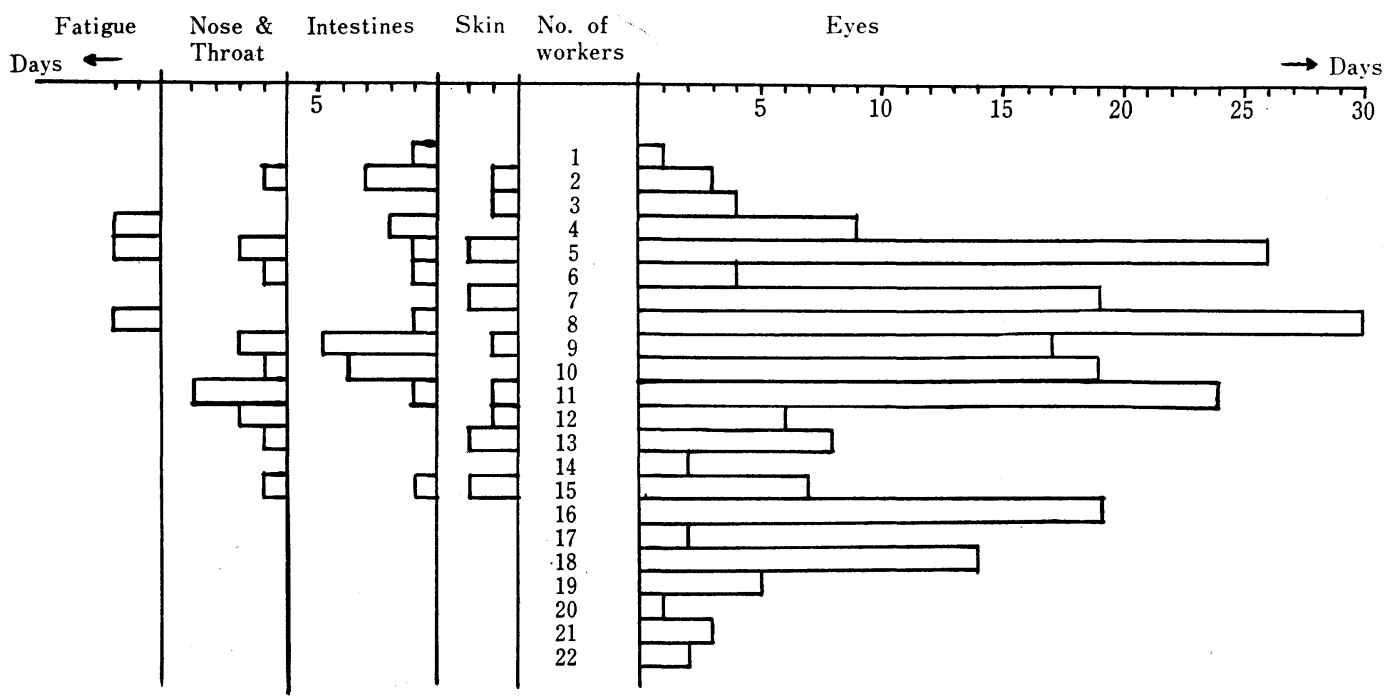

Fig. 4. Days of treatment (on each impairment).

\section{Period of Medical Treatment for Each Type of Injury}

Period required for medical treatment according to the site of injury is illustrated in Fig. 4, which shows the medical treatment received by workers during the production of Blasticidin-S from December 1961 to April 1962. The examination was made with workers in the crystallization and drying processes during the work. This graph shows the total number of days required for treatment and shows that the period is varied, some receiving a long period of treatment after one exposure to the antibiotic, while others experienced only slight injury even after being exposed to the antibiotic 
several times. Eye treatment was the most frequent. The small number of diarrhoea cases is probably due to the absence of abdominal pain and the workers need no medical treatment.

\section{DISCUSSION AND CONCLUSION}

It may be said from clinical cases mentioned above that Blasticidin-S has a obvious inflammatory effect, because the majority of above complaints are symptoms of acute inflammation of body surface exposed to the antibiotic. However, the epidermis itself is comparatively resistant to Blasticidin-S and the antibiotic seems to enter through the sweat and sebaceous glands. Symptomatically, the effect differed from that of caustic agents like acids and alkalines. Inflammation and rash of the skin, and diarrhoea generally appeared a few hours to 24 hours after handling the antibiotic, and appearance of symptoms after the entrance of Blasticidin-S is from 4 to 10 hours in all instances. The marked inflammation caused by the antibiotic results in further reaction of the body, and this seems to be followed by rapid restoration and cure. Repeated exposure to Blasticidin-S seems to make the symptoms chronic but skin injury did not seem to result in adaptation by continued work.

Immediate measures to be taken against this state of affairs is to minimize exposure to Blasticidin-S by improvement of working conditions and environment. From February 1962, 'resting' period system has been adopted and each worker was decided to be kept away from work for an average of $4 \sim 5$ days per month. This has prevented continuous exposure to the antibiotic and decreased the number of injuries. A new factory is now being built and working conditions and facilities will be improved further.

For medical measures against individual workers, vitamin preparation was given to all workers in order to supplement nutrition- and vitamin-deficiency symptoms due to diarrhoea. Washing of eyes was being enforced and eye lotion of antibiotics and adrenocortical steroids were given in the case of erosion and ulceration, which have enhanced recovery. As diarrhoea was different from that caused by bacillary infection or indigestion and was thought to be a kind of reactive diarrhoea, any drug treatment was not given.

For preventive measure, alminium silicate preparation was recommended for the protection of mucous membrane but almost none of the workers followed this recommendation. Most of the skin rash was cured by the application of zinc oxide-olive oil mixture and the use of antiphlogistic and antibiotics for suppurative cases. For damages to mucous membrane of the nose and throat, dexamethazone spray proved to be very effective.

\section{ACKNOWLEDGEMENT}

The author expresses her deepest gratitude to Professor Haruo Katsunuma and 


\section{UNFAVOURABLE EFFECT OF BLASTICIDIN-S}

Assistant-Professor Akira Koizumi, Department of Public Health, University of Tokyo, for their unfailing and kind guidance throughout the course of the present work. She is indebted to Dr. Sumiko Ishizu for her valuable advices, to Dr. O. Yokota, Department of Internal Medicine, University of Tokyo, to Professor Y. Uchida, Department of Ophthalmology, University of Tokushima, for their kind co-operation, and to Mr. Suzuki of K Company for his kind support to the author.

\section{REFERENCES}

1) Takeuchi, S., Hirayama, K., Ueda, K., Sakai, H. and Yonehara, H. (1958). J. Antibiotics (Tokyo), Ser. A, 11, 1.

2) Sakagami, Y. (1961). J. Antibiotics (Tokyo), Ser. A, 14, 247. 